\title{
Renaissance of Chlorthalidone
}

\author{
${ }^{1}$ Anil Pareek, ${ }^{2}$ Mahesh Abhyankar, ${ }^{3}$ Suraj Ghag
}

\section{ABSTRACT}

Hypertension is the major cardiovascular (CV) risk factor and remains inadequately treated in most populations. Thiazides have been the mainstay of hypertension treatment, either as monotherapy or in combination with other antihypertensives.

The most overwhelming evidences of blood pressure (BP) reduction, CV risk reduction, stroke reduction and mortality reduction are demonstrated with chlorthalidone (CTD), established through many landmark studies including the latest SPRINT trial. Chlorthalidone has a longer elimination half life and is two-times more potent than hydrochlorothiazide (HCTZ). The 24 hours BP control, specially late-night to early-morning control is better with CTD compared to HCTZ.

The American Diabetes Association recommends various drugs including a thiazide diuretic to achieve BP targets. Despite a less favorable metabolic profile, initial therapy with thiazide-like diuretics offers similar, and in some instances possibly superior CV outcomes in older hypertensive adults with metabolic syndrome, as compared to treatment with calcium channel blockers (CCB) and angiotensin converting enzyme inhibitor (ACE-I).

Hypokalemia is the major concern associated with CTD use, probably because of use of high doses $>25 \mathrm{mg}$. Use of low-dose CTD, especially $6.25 \mathrm{mg}$, is not associated with any significant change in potassium and sodium levels. This further reduces the risk of new-onset diabetes. Published Indian evidence indicates that use of low-dose of CTD $(6.25$ $\mathrm{mg}$ ) could reduce dose-related concerns about metabolic adverse effects.

Thiazide-type diuretics offer added beneficial effects in terms of reduced risk of hip and pelvic fractures in elderly. ALLHAT and SHEP study have demonstrated that development of incident diabetes with CTD did not have significant associations with CV mortality rate or total mortality rate.

In conclusion, the risk of diabetes associated with CTD should not discourage clinicians from using it long-term to reduce $\mathrm{CV}$ risks. $\mathrm{CV}$ risk reduction remains the ultimate goal of any antihypertensive therapy and the beneficial effects of CTD remain unsurpassed in this aspect.

Keywords: Hypertension, Chlorthalidone, Diuretics, Thiazides.

How to cite this article: Pareek A, Abhyankar M, Ghag S. Renaissance of Chlorthalidone. Hypertens J 2016;2(4):200-208.

Source of support: Nil

Conflict of interest: Authors are employed with IPCA laboratories Ltd. in research work of Chlorthalidone.

\footnotetext{
${ }^{1}$ President, ${ }^{2}$ General Manager, ${ }^{3}$ Manager

${ }^{1-3}$ Medical Affairs and Clinical Research, IPCA Laboratories Limited, Mumbai, Maharashtra, India
}

Corresponding Author: Anil Pareek, President, Medical Affairs and Clinical Research, IPCA Laboratories Limited, Mumbai Maharashtra, India, e-mail: anil.pareek@ipca.com

\section{HYPERTENSION: DISEASE BURDEN}

Hypertension is a major cardiovascular (CV) risk factor, but the real magnitude of the problem and its determinants are still not fully understood. Although there has been widespread dissemination of knowledge about hypertension, it remains inadequately treated in most populations including in India. ${ }^{1}$ It is estimated that one of four adults in the urban population has a blood pressure (BP) reading exceeding $140 / 90 \mathrm{~mm} \mathrm{Hg} .{ }^{2}$ Significant number of individuals with hypertension are unaware of their condition. ${ }^{3}$ Even among persons identified as being hypertensive, only about half are being currently treated; and among patients receiving drug treatment, only half have their BP fully normalized. ${ }^{4}$ Hypertension is often accompanied by other conditions, such as impaired glucose homeostasis (diabetes and prediabetes) and dyslipidemia often as part of metabolic syndrome. ${ }^{5}$

\section{Thiazide Diuretics in Hypertension}

Thiazide diuretics have been the mainstay of hypertension treatment for last five decades, either given as monotherapy or used in combination with other antihypertensive drugs. Clinical experts and guidelines are now suggesting preferential use of thiazide-like diuretics over thiazide diuretic. ${ }^{6,7}$ The purpose of treating hypertension is not only to decrease BP but also to decrease $\mathrm{CV}$ morbidity and mortality. Cardiovascular risk reduction, stroke reduction, and mortality reduction evidence are overwhelming with chlorthalidone (CTD) ${ }^{8-10}$

National and international guidelines have recommended using thiazide-like diuretics as first-line treatment for hypertension. ${ }^{11,12}$ A Scientific Statement from the AHA/ACC/ASH on "Treatment of hypertension in patients with coronary artery disease" recommends CTD as a preferred diuretic in patients with stable angina, acute coronary syndrome (ACS), and heart failure (HF). ${ }^{11}$ The National Institute for Health and Care Excellence Hypertension Clinical guideline for adults in diagnosis and management recommends that if diuretic treatment is to be initiated or changed, offer a thiazide-like diuretic, such as CTD or indapamide in preference to a conventional thiazide diuretic, such as bendroflumethiazide or hydrochlorothiazide (HCTZ). ${ }^{12}$ The Indian Guidelines on Hypertension recommend CTD is to be preferred over HCTZ as an antihypertensive. ${ }^{7}$ 


\section{Comparison with HCTZ}

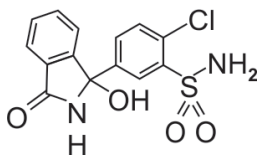

Chlorthalidone belongs to monosulfonamyl diuretic group, being known for long as thiazide-related diuretic. ${ }^{13}$ Chlorthalidone is a structurally and pharmacokinetically distinct compound to HCTZ with a much longer half-life for effect and a wider volume of distribution with heavy partitioning in red blood cells. These features afford CTD a unique advantage in its capacity to act as an effective diuretic and BP lowering agent, as well as a compound that improves $\mathrm{CV}$ outcomes in the patient with hypertension. ${ }^{14}$

Chlorthalidone is two times more potent than HCTZ, so $6.25 \mathrm{mg}$ of CTD is equivalent to $12.5 \mathrm{mg}$ of HCTZ. ${ }^{15}$ Chlorthalidone has a longer elimination of half-life, 45 to 60 hours, than HCTZ of 8 to 15 hours. The antihypertensive action of CTD may last for 48 to 72 hours. ${ }^{16}$

In the study conducted by Ernst et $\mathrm{al}^{17}$ after 8 weeks, patients who were taking CTD experienced a greater reduction in $\mathrm{BP}$ than those taking $\mathrm{HCTZ}$. The data indicated a greater reduction in 24-hour mean systolic blood pressure (SBP) with CTD compared with HCTZ (Table 1).

Late night to early morning, BP control is poor with HCTZ. Importantly, this time period has been identified as the most critical for CV events. ${ }^{18}$

\section{Mechanism of BP Control with CTD}

Hypotensive effect of CTD in hypertensive persons for a brief period is initially due to a reduction in the plasma volume. Initial action for 4 to 6 weeks is by lowering cardiac output. Later, cardiac output comes to normal, but $\mathrm{BP}$ remains low, which is due to reduction in peripheral vascular resistance (PVR). Sustained action is probably due to its direct action on arterioles. Chlorthalidone can reduce $\mathrm{BP}$ by multiple ways, such as reduction in plasma volume, reduction in PVR by interference with intracellular $\mathrm{Ca}^{2+}$ release by noradrenaline, inhibition of rho kinase activity, reduction in arterial edema, and reduction in vascular reactivity. ${ }^{19-23}$

\section{Major Global Clinical Trials of CTD}

The BP lowering efficacy and reduction in CV events by CTD is established in many National Institute of Health (NIH) sponsored studies, including the latest SPRINT trial. TOMHS - Treatment of Mild Hypertension Study

Table 1: Change in 24-hour mean and nighttime BP with CTD and HCTZ

\begin{tabular}{lll}
\hline & 24-Hour mean & \\
Drug & $B P(\mathrm{~mm} \mathrm{Hg})$ & Nighttime $B P(\mathrm{~mm} \mathrm{Hg})$ \\
\hline CTD $25 \mathrm{mg} /$ day & $-12.4 \pm 1.8$ & $-13.5 \pm 1.9 \mathrm{~mm}$ of Hg \\
HCTZ $50 \mathrm{mg} /$ day & $-7.4 \pm 1.7$ & $-6.4 \pm 1.8 \mathrm{~mm}$ of Hg \\
\hline
\end{tabular}

MRFIT - Multiple Risk Factor Intervention Trial SHEP - Systolic Hypertension in the Elderly Program ALLHAT - Antihypertensive and Lipid-Lowering Treatment to Prevent Heart Attack Trial

SPRINT - Systolic Blood Pressure Intervention Trial

\section{TREATMENT OF MILD HYPERTENSION STUDY24}

Six antihypertensive interventions for the treatment of mild hypertension [diastolic BP (DBP) $<100 \mathrm{~mm} \mathrm{Hg}$ ] were evaluated in a randomized, double-blind, placebocontrolled clinical trial. Participants randomly received placebo $(n=234)$, CTD $(n=136)$, acebutolol $(n=132)$, doxazosin $(n=134)$, amlodipine $(n=131)$, or enalapril $(n=135)$. Blood pressure reduction in the drug treatment group $(15.9 / 12.3 \mathrm{~mm} \mathrm{Hg})$ was significantly more than the placebo group $(9.1 / 8.6 \mathrm{~mm} \mathrm{Hg})$. Less number of participants in the drug treatment groups died or experienced a major nonfatal $\mathrm{CV}$ event than those assigned to the placebo group (5.1 vs 7.3\%). There was a significant decrease in all treatment groups (10-15\%) in left ventricular mass (LVM) from baseline and continued for 48 months. The CTD group demonstrated the greatest decrease in LVM (mean decrease $=34 \mathrm{gm}$ ) compared with other groups (mean decrease $=24-27 \mathrm{gm}$ ).

\section{MULTIPLE RISK FACTOR INTERVENTION TRIAL ${ }^{10}$}

The Multiple Risk Factor Intervention Trial (MRFIT) included 12,866 high-risk men aged 35 to 57 years in 1972 at 28 institutes. They were assigned to a special intervention program that included lifestyle modifications or controlled group. At some centers, special intervention group received a diuretic ( 9 centers HCTZ and 6 centers CTD).

The HCTZ group had a $44 \%$ higher mortality. Hence, the patients were shifted to CTD. Later with CTD the trend was reversed and the same group had a $28 \%$ lower risk.

\section{SYSTOLIC HYPERTENSION IN THE ELDERLY PROGRAM ${ }^{9}$}

In a multicentric, double-blind, placebo-controlled trial, 4,736 persons aged 60 years and above with SBP from 160 to $219 \mathrm{~mm} \mathrm{Hg}$ and DBP $<90 \mathrm{~mm} \mathrm{Hg}$ were randomized (2,365 to CTD, $12.5-25 \mathrm{mg} /$ day and 2,371 to placebo). After 1 year, $83 \%$ of the CTD group and $80 \%$ of the placebo group were still taking medications. Of those still taking CTD, $88 \%$ had reached goal BP without requiring a step II drug and most had responded to $25 \mathrm{mg} /$ day. The mean difference between the two groups was $17 \mathrm{~mm} \mathrm{Hg}$ for SBP $(\mathrm{p}<0.001)$ and $6 \mathrm{~mm} \mathrm{Hg}$ for DBP $(\mathrm{p}<0.001)$. The 5 -year average $\mathrm{BP}$ was $155 / 72 \mathrm{~mm} \mathrm{Hg}$ for the placebo group and $143 / 68 \mathrm{~mm} \mathrm{Hg}$ for the active treatment group. The 5-year incidence of total stroke was 5.2 per 100 participants for active treatment and 8.2 per 100 for placebo. The relative 
risk with active treatment group was 0.64 for stroke, 0.73 for clinical nonfatal myocardial infarction (MI) plus coronary death, 0.68 for major CV events, and 0.87 for deaths from all causes. This study indicated that CTD was effective for lowering BP in elderly patients with systolic hypertension. In patients with isolated systolic hypertension, treatment with CTD as step 1 drug decreased the incidence of total stroke by $36 \%$. The 5 -year absolute benefit was 30 stroke events and $55 \mathrm{CV}$ events per 1,000 participants.

\section{SYSTOLIC HYPERTENSION IN THE ELDERLY PROGRAM EXTENDED ${ }^{25}$}

At the 22-year follow-up of SHEP, life expectancy gain, expressed as the area between active $(n=2,365)$ and placebo $(n=2,371)$ survival curves, was 105 days for all-cause mortality and 158 days for cardiovascular disease (CVD) death . Each month of active treatment was therefore associated with approximately 1 day extension in life expectancy. The active treatment group had higher survival free period from $C V$ death vs the placebo group $(p=0.03)$, but similar survival for all-cause mortality. Thus in the SHEP trial, treatment of isolated systolic hypertension with CTD stepped-care therapy for 4.5 years was associated with longer life expectancy at 22 years of follow-up.

\section{ANTIHYPERTENSIVE AND LIPID- LOWERING TREATMENT TO PREVENT HEART ATTACK TRIAL ${ }^{8}$}

The ALLHAT was a randomized, double-blind study in a total of 42,418 participants aged $\geq 55$ years with hypertension and at least 1 other coronary heart disease (CHD) risk factor. Participants received CTD, 12.5 to $25 \mathrm{mg}$ /day $(\mathrm{n}=15,255)$, amlodipine, 2.5 to $10 \mathrm{mg} /$ day $(\mathrm{n}=9,048)$, lisinopril, 10 to $40 \mathrm{mg} /$ day $(\mathrm{n}=9054)$; or doxazocin $(\mathrm{n}=$ 9061) for 4 to 8 years. The doxazosin arm was stopped early due to a $25 \%$ higher rate of combined CVD and a two times higher rate of HF compared with the CTD arm. At 5 years, the mean BP was 133.9/75.4 mm Hg in the CTD group, 134.7/74.6 $\mathrm{mm} \mathrm{Hg}$ in the amlodipine group, and 135.9/75.4 in the lisinopril group. Also at 5 years, the percentage of patients who had achieved the goal BP $(<140 / 90 \mathrm{~mm} \mathrm{Hg}$ ) was $68.2 \%$ in the CTD group, $66.3 \%$ in the amlodipine group, and $61.2 \%$ in the lisinopril group.

The amlodipine group had a 38\% higher risk of HF ( $p<0.001)$ and a $35 \%$ higher risk of hospitalized/fatal HF $(\mathrm{p}<0.001)$ compared to CTD. There was no significant difference observed between amlodipine and CTD groups for combined outcome of fatal CHD or nonfatal MI, allcause mortality, combined CHD, stroke, combined CVD, angina, coronary revascularization, peripheral arterial disease, cancer, or end-stage renal disease (ESRD). The lisinopril group had a $15 \%$ higher risk of stroke $(\mathrm{p}=0.02)$, $10 \%$ higher risk of combined CVD ( $p<0.001), 19 \%$ higher risk of $\mathrm{HF}(\mathrm{p}<0.001)$, and $11 \%$ higher risk of hospitalized or treated angina $(p=0.01)$. There was no significant difference observed between amlodipine and CTD groups for combined outcome of fatal CHD or nonfatal MI, all-cause mortality, combined CHD, hospitalized/fatal HF, hospitalized angina, coronary revascularization, peripheral arterial disease, cancer, or ESRD. Chlorthalidone was superior to amlodipine and lisinopril in HF prevention during the first year of follow-up, with a $>50 \%$ reduction in incidence. Beyond year 1, CTD was superior to amlodipine and equivalent to lisinopril in HF prevention. These results held true when examined by subgroups of age, race, sex, and diabetes status. Diuretics are clearly preferred over calcium channel blockers (CCBs) overall and over angiotensin converting enzyme (ACE) inhibitors, at least in the short-term, in preventing HF.

The higher potency of CTD resulted in longer duration of action that provided nighttime BP control and hence, was effective in providing additional protection from stroke and MI during early morning BP surge.

\section{SYSTOLIC BLOOD PRESSURE INTERVENTION TRIAL ${ }^{26}$}

The most appropriate targets for SBP to reduce CV morbidity and mortality among persons without diabetes were determined in a randomized controlled trial (RCT). A total of 9,361 persons with a SBP of $\geq 130 \mathrm{~mm} \mathrm{Hg}$ and an increased $\mathrm{CV}$ risk, but without diabetes, were randomized to a SBP target $<120 \mathrm{~mm} \mathrm{Hg}$ (intensive treatment) or $<140 \mathrm{~mm} \mathrm{Hg}$ (standard treatment). Use of drug classes with the strongest evidence for reduction in $\mathrm{CV}$ outcomes, including thiazide-type diuretics (encouraged as the firstline agent), loop diuretics (for participants with advanced chronic kidney disease), and beta-adrenergic blockers (for those with coronary artery disease) was encouraged.

Chlorthalidone was encouraged as the primary thiazide-type diuretic, and amlodipine as the preferred CCB. At 1 year, the mean SBP was $121.4 \mathrm{~mm} \mathrm{Hg}$ in the intensive treatment group and $136.2 \mathrm{~mm} \mathrm{Hg}$ in the standard-treatment group. The intervention was stopped early after a median follow-up of 3.26 years, owing to a significantly lower rate of the primary composite outcome (first occurrence of a MI, ACS, stroke, HF, or CVD death) in the intensive-treatment group than in the standard-treatment group. The intensive-treatment group participants had a $25 \%$ lower relative risk of primary outcome, $38 \%$ lower relative risk of $\mathrm{HF}, 43 \%$ lower relative risk of death from $\mathrm{CV}$ causes, and $27 \%$ lower relative risk of death from any cause.

\section{Diabetic Hypertension and Use of CTD}

In the Systolic Hypertension in the Elderly Program (SHEP) study, a total of 4,736 men and women aged 60 years and older with SBP $\geq 160 \mathrm{~mm} \mathrm{Hg}$ were included. 
Out of which, 583 had noninsulin-dependent diabetes mellitus (NIDDM) and 4,149 were nondiabetic. Rates of CVD events and of all-cause mortality were higher for diabetic than nondiabetic persons, but lower for both active treatment groups compared with the placebo groups. ${ }^{9}$

For diabetic patients randomized to SHEP active treatment of CTD compared with diabetic patients randomized to placebo, rates were lower for major $\mathrm{CV}$ events, stroke, nonfatal MI plus fatal CHD, major coronary CHD events, and all-cause mortality. Results with SHEP antihypertensive treatment were similar for diabetic and nondiabetic patients and, in some cases, more favorable for diabetic patients. Since diabetic patients had higher rates than nondiabetic patients, treatment-attributable reduction in absolute risk was greater for diabetic patients. ${ }^{9}$
Chlorthalidone treatment prevented 101 diabetic participants per 1,000 from having a CVD event, compared with 51 per 1,000 nondiabetic participants, i.e., twice as great a reduction in absolute risk. This can be seen in the graph above showing absolute risk reduction. ${ }^{9}$

American Diabetes Association Standards of Medical Care in Diabetes 2015 recommend various drugs including a thiazide diuretic to achieve BP targets. ${ }^{27}$

The metabolic syndrome is a clustering of clinical and biochemical characteristics related to insulin resistance. It is characterized by hypertension, central obesity, dyslipidemia (high triglycerides, low high-density lipoprotein cholesterol levels), and elevated glucose levels. To date, there is no consensus as to which class of antihypertensive medications, if any, is preferred for the treatment of
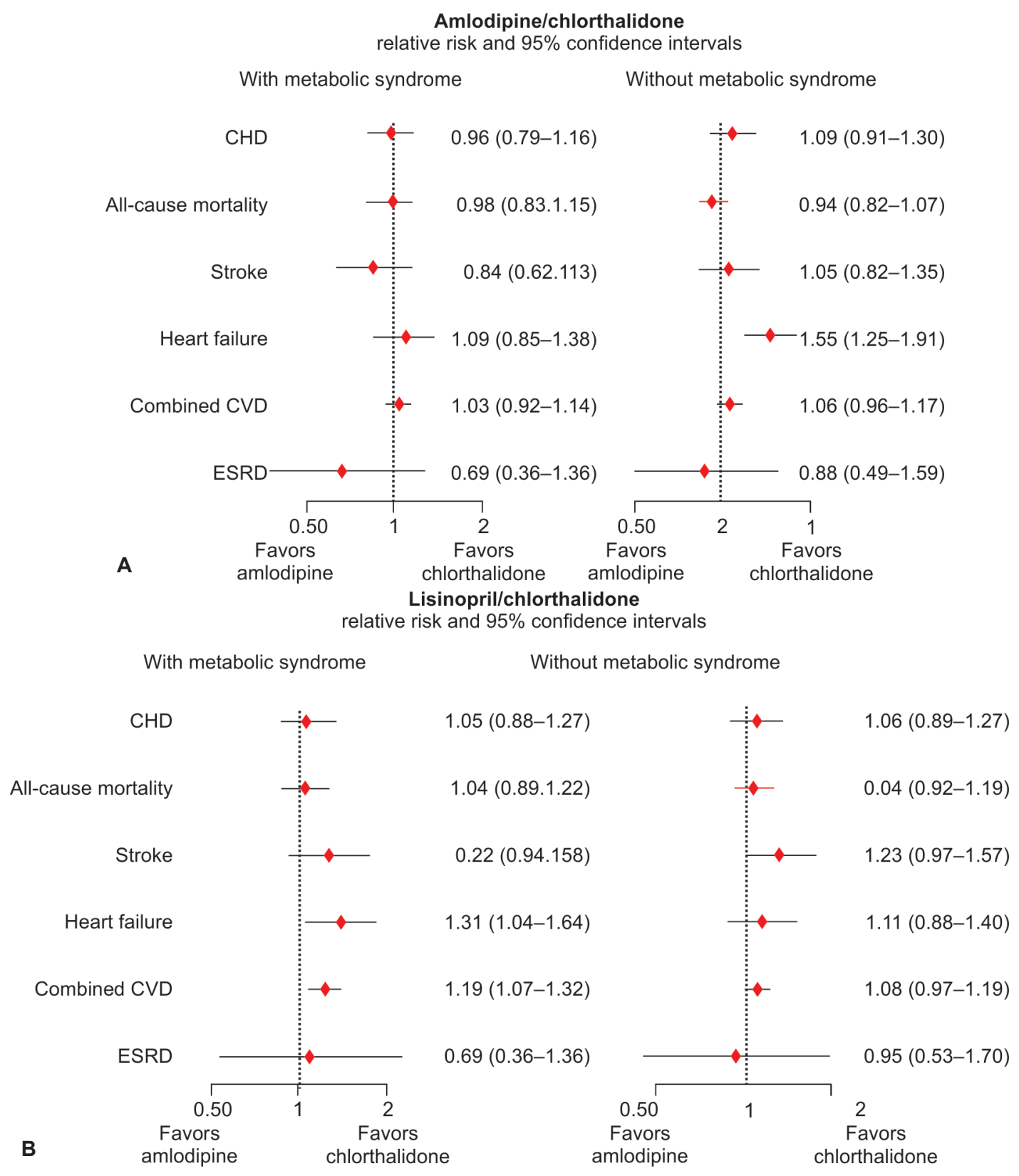

Figs $1 \mathrm{~A}$ and B: Antihypertensive and lipid-lowering treatment to prevent heart attack trial subgroup analysis in metabolic syndrome 
hypertension in patients with the metabolic syndrome. These issues were addressed in a subgroup analysis of the Antihypertensive and Lipid-Lowering Treatment to Prevent Heart Attack Trial (ALLHAT). The results are shown in Figure $1 \mathrm{~A}$ and B. ${ }^{28}$

Despite a less favorable metabolic profile, thiazidelike diuretic for hypertension offers similar, and in some instances possibly superior, CVD outcomes in older hypertensive adults with metabolic syndrome, as compared to treatment with CCBs and ACE inhibitors.

\section{Role of Chlorthalidone $6.25 \mathrm{mg}$}

Pareek et $\mathrm{al}^{29}$ compared the efficacy and safety of low-dose CTD + atenolol combination with atenolol and atenolol + amlodipine combination in stage I hypertensive patients uncontrolled on active run-in monotherapy. Newly diagnosed stage I hypertensive patients were randomized to active run-in monotherapy either with atenolol $25 \mathrm{mg}$ (98/300) or CTD $6.25 \mathrm{mg}(100 / 300)$ or amlodipine $2.5 \mathrm{mg}$ (102/300). A total of 282/300 patients (atenolol 92, CTD 91, amlodipine 99) completed the active run-in phase successfully. The mean fall in SBP and DBP was comparable for study treatments at week 12 and at week 20; also, the percentage of responders was comparable for the three study treatment groups indicating that the low-dose CTD + atenolol combination is noninferior to the high-dose atenolol alone and atenolol+amlodipine combination.

Pareek et $\mathrm{al}^{30}$ evaluated the efficacy of metoprolol XL/ CTD against metoprolol XL/HCTZ with respect to mean fall in SBP and DBP. In this randomized, comparative, multicentric, 12-week study, 130 eligible patients (65: metoprolol XL $25 \mathrm{mg} / \mathrm{CTD} 6.25 \mathrm{mg}$; 65: metoprolol XL $25 \mathrm{mg}$ /HCTZ $12.5 \mathrm{mg}$ ) were enrolled. Sixty-two patients from each group completed the study. Both the starting therapies were comparable with respect to mean fall in SBP and DBP and response rates after 4 weeks of therapy. Also both the step-up therapies showed similar mean fall in SBP and DBP at the end of 12 weeks. There were no clinically significant trends in electrolytes $\left(\mathrm{Na}^{+}, \mathrm{K}^{+}, \mathrm{Cl}^{-}\right)$ and fasting blood sugar, that was evident across the treatment groups. Chlorthalidone in combination with metoprolol XL was as effective and well tolerated as widely used combination of metoprolol XL/HCTZ, thus providing an alternative therapeutic option.

Pareek et $\mathrm{al}^{15}$ evaluated the antihypertensive efficacy of losartan/CTD vs losartan/HCTZ in mild-to-moderate essential hypertension. In that study 131 patients were randomized to losartan $25 \mathrm{mg} / \mathrm{CTD} 6.25 \mathrm{mg}(\mathrm{n}=66)$ or to losartan $25 \mathrm{mg} / \mathrm{HCTZ} 12.5 \mathrm{mg}(\mathrm{n}=65)$. After 4 weeks of therapy, both treatments showed a significant fall from baseline in SBP/DBP (-20.17/-10.30 mm Hg vs $-17.63 /-10.20 \mathrm{~mm} \mathrm{Hg}$ ). Both treatments were similar with respect to mean fall in SBP, DBP, and response rate. The losartan/low-dose CTD (6.25 mg) combination is as effective as the widely used losartan/HCTZ combination in lowering BP and is well tolerated, thus providing a useful therapeutic option for treating mild-to-moderate hypertension.

Pareek et $\mathrm{al}^{18}$ recently published a 12-week comparative, double-blind, outpatient RCT. The study randomized 54 patients with stage 1 hypertension to receive either CTD $6.25 \mathrm{mg}$ ( $\mathrm{n}=16)$; HCTZ $12.5 \mathrm{mg}(\mathrm{n}=18)$; or HCTZ-CR $12.5 \mathrm{mg}(\mathrm{n}=20)$. All three treatments significantly ( $\mathrm{p}<0.01$ ) lowered office BP at weeks 4 and 12 from baseline. At weeks 4 and 12, significant reductions in systolic and diastolic 24 hours ambulatory $\mathrm{BP}(\mathrm{ABP})$ and nighttime BP $(\mathrm{p}<0.01)$ were observed with CTD but not with HCTZ. At weeks $4(p=0.015)$ and $12(p=0.020)$, nighttime systolic ABP was significantly lower in the CTD group than in the HCTZ group. In contrast to the HCTZ group, the HCTZ-CR group also showed a significant $(\mathrm{p}<0.01)$ reduction in 24-hour ABP. Treatment with low-dose CTD, $6.25 \mathrm{mg}$ daily, significantly reduced mean 24-hour ABP as well as daytime and nighttime BP. Due to its short duration of action, no significant 24-hours ABP reduction was seen with HCTZ, 12.5 mg daily, which merely converted sustained hypertension into masked hypertension. Thus, low-dose CTD, $6.25 \mathrm{mg}$, could be used as monotherapy for treatment of essential hypertension, whereas low-dose HCTZ monotherapy was not an appropriate antihypertensive drug (Graph 1 ).

In trials conducted on Indian patients, the effect on electrolyte levels with CTD was seen ${ }^{15,29,30}$ (Table 2).

\section{Hypokalemia and Hyponatremia}

Concerns about greater loss of potassium with the use of CTD has always averted clinicians from using this drug. However, this fear was possibly influenced by the

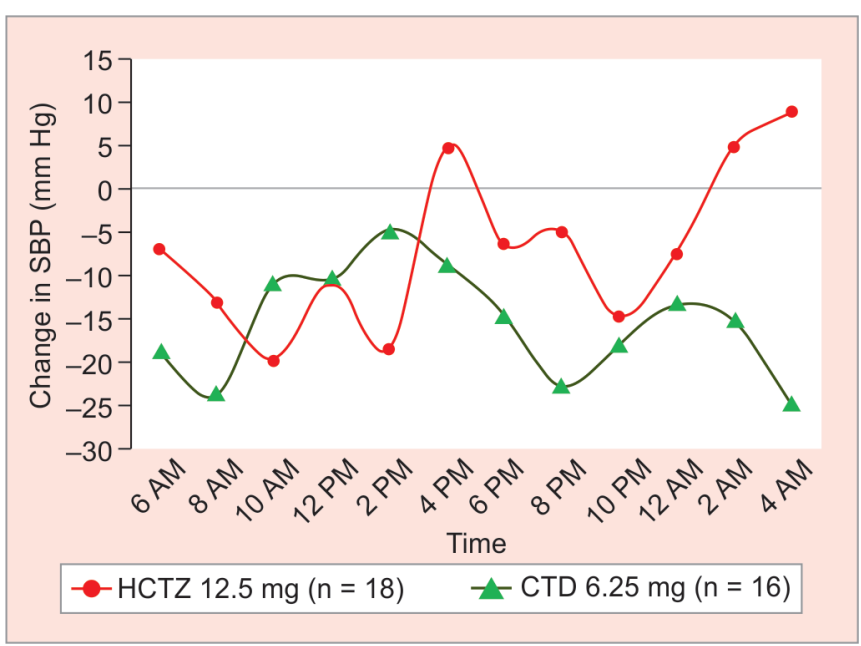

Graph 1: Mean change from baseline to week-12 in average ambulatory systolic BP 
Table 2: Effect of chlorthalidone on electrolyte levels

\begin{tabular}{llll}
\hline $\begin{array}{l}\text { SI. } \\
\text { no. }\end{array}$ & Nature of study & $\begin{array}{l}\text { No. of pts } \\
\text { duration }\end{array}$ & Conclusions for electrolyte imbalance \\
\hline 1 & Low-dose CTD with atenolol & 300 pts & Low-dose CTD for up to 6 months, either alone or in combination, \\
& & 20 weeks & had no reports of electrolyte imbalance in the studied population. \\
2 & Metoprolol XL/CTD low-dose vs Metoprolol & 130 pts & Data collected from this study did not report any clinically \\
& XL/HCTZ & significant adverse metabolic events. \\
3 & Losartan-low dose CTD vs losartan-HCTZ & 131 pts & Changes in serum electrolytes were clinically unremarkable \\
& & 12 weeks & across the therapy groups.
\end{tabular}

Note: In susceptible patients one needs to keep a watch on fluid or electrolyte imbalance

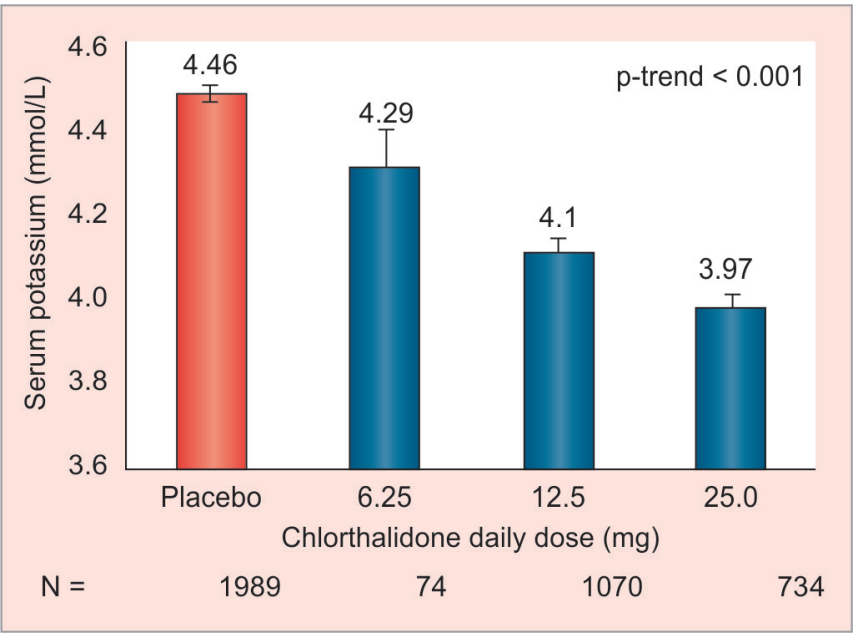

Graph 2: Potassium levels in SHEP study with different doses of CTD (Source: Franse LV, et al. Hypertension 2000 May;35(5):1025-1030)

historical use of higher doses of the drug (50-100 mg) than now typically used. As long as very high doses of CTD are not used, the metabolic issues particularly of hypokalemia should not be a problem (Graph 2).

When low-dose CTD was used in Indian patients, the change in serum potassium levels and serum sodium was not significant (Table 3).

In the ALLHAT study, the change in potassium levels did not translate into more $\mathrm{CV}$ events or into higher allcause mortality in the CTD group compared with the other groups of lisinopril or amlodipine. ${ }^{8}$ In the SHEP trial, during follow-up, the mean sodium concentrations was similar in the active treatment group and placebo group. ${ }^{9}$

\section{Hyperglycemia}

Pancreatic release of insulin is controlled via adenosine triphosphate-sensitive potassium channels and L-type calcium channels on the beta-cell surface. Increase in plasma glucose closes the potassium channels and increases insulin secretion. Changes in serum potassium may prevent closure of these channels leading to decrease in insulin secretion. Hypertension is often associated with insulin resistance. In the presence of insulin resistance, decrease in beta-cell insulin release due to changes in potassium may lead to hyperglycemia in individuals with insulin resistance. ${ }^{31}$

Thiazide-induced diabetes occurs early after initiating therapy treatment. Nondiabetic patients currently on thiazide therapy for greater than 1 year are unlikely to develop thiazide-induced diabetes. Chlorthalidoneinduced diabetes appeared to be mediated by changes in serum potassium. Each $0.5 \mathrm{mEq} / \mathrm{L}$ decrease in serum potassium from the baseline during year 1 was associated with a $45 \%$ higher risk of diabetes. Treatment with CTD doses $>12.5 \mathrm{mg} /$ day is an important risk factor. ${ }^{31}$

From the SHEP trial it was seen that fasting glucose levels increased in both groups. The incidence of diabetes was studied in CTD treated group and placebo. New cases of diabetes were $3.6 \%$ in CTD group and $2.9 \%$ in placebo at 1 year. At 3 years the incidence of diabetes was 8.6\% in CTD and 7.5\% in placebo. These differences are not statistically significant. ${ }^{9}$

Indian studies done by Pareek et al $1^{15,29,30}$ showed the following changes in blood glucose levels (Table 4).

Table 3: Effect of low-dose CTD $(6.25 \mathrm{mg})$ on potassium and sodium levels

\begin{tabular}{llll}
\hline & Atenolol $25 \mathrm{mg}+$ & Metoprolol XL 25 mg + & Losartan 25 mg + \\
Laboratory parameter $(\mathrm{mEq} / \mathrm{L})$ & Chlorthalidone $6.25 \mathrm{mg}$ & Chlorthalidone 6.25 mg & $4.24 \pm 0.40$ \\
\hline Baseline serum potassium & $4.21 \pm 0.51$ & $4.16 \pm 0.46$ & $3.99 \pm 0.55$ \\
End of study serum potassium & $4.16 \pm 0.40$ & $3.99 \pm 0.55$ & $139.27 \pm 3.68$ \\
Baseline serum sodium & $139.53 \pm 3.65$ & $137.84 \pm 3.45$ & $138.70 \pm 2.40$ \\
End of study serum sodium & $139.45 \pm 3.24$ & $139.59 \pm 4.33$ & $\mathrm{mg}$ \\
\hline
\end{tabular}

Table 4: Effect of low-dose CTD on fasting plasma glucose levels

\begin{tabular}{llll}
\hline & Atenolol $25 \mathrm{mg}+$ & Metoprolol XL 25 mg + & Losartan 25 mg + \\
Fasting blood glucose $(\mathrm{mg} / \mathrm{dL})$ & Chlorthalidone $6.25 \mathrm{mg}$ & Chlorthalidone 6.25 mg & Chlorthalidone 6.25 mg \\
\hline Baseline & $100.76 \pm 22.24$ & $111.98 \pm 40.20$ & $114.67 \pm 33.43$ \\
End of study & $100.38 \pm 16.01$ & $104.71 \pm 26.49$ & $108.95 \pm 28.49$ \\
\hline
\end{tabular}


Thus, Indian studies have not shown cause for concern as regard to glucose levels for the duration of studies.

In a post hoc subgroup analysis in nondiabetic participants from ALLHAT who were randomized to receive treatment with CTD $(n=8,419)$, amlodipine $(n=4,958)$ or lisinopril $(n=5,034)$ and observed for a mean of 4.9 years. Fasting glucose levels increase in older adults with hypertension regardless of treatment type. For those taking CTD vs other medications, the risk of developing fasting blood glucose levels higher than $125 \mathrm{mg} / \mathrm{dL}$ is modestly greater, but there is no conclusive or consistent evidence that this diuretic-associated increase in diabetes mellitus risk increases the risk of clinical events. ${ }^{32}$

\section{BENEFITS OF CTD IN PATIENTS WITH PREEXISTING AND NEW-ONSET DIABETES}

The SHEP collaborative research group studied the longterm effects of diuretic-based therapy on fatal outcomes in subjects with isolated systolic hypertension with and without diabetes. ${ }^{33}$ At a mean follow-up of 14.3 years, CV mortality rate was significantly lower in the CTD group $(19 \%)$ than in the placebo group (22\%). Diabetes that developed during trial in placebo treated patients $(n=169)$ was associated with increased CV adverse outcome and total mortality rate. However, diabetes that developed among patients during diuretic therapy did not have significant associations with CV mortality rate or total mortality rate.

Diuretic treatment in subjects who had diabetes was strongly associated with lower long-term CV mortality rate and total mortality rate.

The CTD based treatment improved long-term outcomes, especially among subjects who had diabetes. Subjects who had diabetes associated with chlorthalidone had no significant increase in CV events and had a better prognosis than did those who had preexisting diabetes.

In the ALLHAT diabetes extension study, ${ }^{34}$ participants on CTD with incident diabetes vs no diabetes had consistently lower, non significant risk for CVD mortality, all cause mortality and non CVD mortality than participants on amlodipine or lisinopril with incident diabetes. Participants with incident diabetes had elevated CHD risk compared with those with no diabetes, but those on CTD had significantly lower risk than those on lisinopril. In conclusion, the findings suggest that thiazide-associated incident DM is associated with lower CVD mortality and incident DM over an average of 6.9 years. Therefore, concerns regarding potentially adverse diabetic effects associated with thiazide-type diuretic therapy should not inhibit its use. In this regard, a recent pooled analysis of 5 statin studies ${ }^{35}$ showed that incident DM was more common in persons treated with intensive-dose therapy versus moderate-dose therapy. Nonetheless, the benefits of reduced cholesterol were deemed to outweigh any possible deleterious effects of incident diabetes mellitus on CVD outcomes. Similarly, thiazide-like diuretics have been shown to be highly effective for preventing CVD outcomes through decades of rigorously controlled clinical trials.

\section{THIAZIDE-LIKE DIURETICS AND REDUCED RISK OF HIP AND PELVIC FRACTURES IN OLDER ADULTS}

The ALLHAT collaborative research group studied the association of 3 different antihypertensive medications with hip and pelvic fracture risk in older adults. ${ }^{36}$ A total of 22,180 participants were followed-up for 8 years. After trial completion, 16,622 participants were followed for additional 5 years. Participants randomized to receive CTD vs amlodipine or lisinopril had a lower risk of fracture on adjusted analysis $(p=0.04)$. Risk of fracture was significantly lower in participants randomized to receive CTD vs lisinopril $(\mathrm{p}=0.04)$. These findings from a large randomized clinical trial provide evidence of a beneficial effect of thiazide-type diuretic therapy in reducing hip and pelvic fracture risk compared with treatment with other antihypertensive medications.

\section{CONCLUSION}

Clinical experts, national, and international guidelines are suggesting preferential use of thiazide like diuretic over thiazide diuretic. Chlorthalidone is a structurally and pharmacokinetically distinct compound to HCTZ with two times more potency and much longer half-life. It ensures effective systolic and diastolic BP reduction in daytime, nighttime, 24-hour ambulatory BP recordings. The renaissance of CTD is due to various global large trials like TOMHS, MRFIT, SHEP, ALLHAT, and SPRINT showing $\mathrm{CV}$ events reduction, stroke reduction, and most importantly, mortality reduction across various patient subgroups.

The dose of CTD $6.25 \mathrm{mg}$ is most suitable for Indian patients. Published Indian evidence indicates that this low dose of CTD could reduce dose-related concerns about metabolic adverse effects and may lead to its wider usage as an antihypertensive agent.

\section{REFERENCES}

1. Anchala R, Kannuri NK, Pant H, Khan H, Franco OH, Di Angelantonio E, Prabhakaran D. Hypertension in India: a systematic review and meta-analysis of prevalence, awareness, and control of hypertension. J Hypertens 2014 Jun;32(6): 1170-1177.

2. Egan BM, Zhao $Y$, Axon RN. US trends in prevalence, awareness, treatment, and control of hypertension, 1988-2008. JAMA 2010 May 26;303(20):2043-2050.

3. Kearney PM, Whelton M, Reynolds K, Whelton PK, He J. Worldwide prevalence of hypertension: a systematic review. J Hypertens 2004 Jan;22(1):11-19. 
4. Wilber JA, Barrow JG. Hypertension - a community problem. Am J Med 1972 May;52(5):653-663.

5. Halpern A, Mancini MC, Magalhães ME, Fisberg M, Radominski R, Bertolami MC, Bertolami A, de Melo ME, Zanella MT, Queiroz MS, et al. Metabolic syndrome, dyslipidemia, hypertension and type 2 diabetes in youth: from diagnosis to treatment. Diabetol Metab Syndr 2010 Aug 18;2:55.

6. Kaplan NM. Chlorthalidone versus hydrochlorothiazide: a tale of tortoises and a hare. Hypertension 2011 Dec;58(6): 994-995.

7. Association of Physicians of India. Indian guidelines on hypertension (I.G.H.) - III. 2013. J Assoc Physicians India 2013 Feb;61(Suppl 2):6-36.

8. ALLHAT Officers and Coordinators for the ALLHAT Collaborative Research Group. The Antihypertensive and LipidLowering Treatment to Prevent Heart Attack Trial. Major outcomes in high-risk hypertensive patients randomized to angiotensin-converting enzyme inhibitor or calcium channel blocker vs diuretic: The Antihypertensive and LipidLowering Treatment to Prevent Heart Attack Trial (ALLHAT). JAMA 2002 Dec 18;288(23):2981-2997.

9. SHEP Cooperative Research Group. Prevention of stroke by antihypertensive drug treatment in older persons with isolated systolic hypertension. Final results of the Systolic Hypertension in the Elderly Program (SHEP). JAMA 1991 Jun 26;265(24):3255-3264.

10. Ernst ME, Neaton JD, Grimm RH Jr, Collins G, Thomas W, Soliman EZ, Prineas RJ, Multiple Risk Factor Intervention Trial Research Group. Long-term effects of chlorthalidone versus hydrochlorothiazide on electrocardiographic left ventricular hypertrophy in the multiple risk factor intervention trial. Hypertension 2011 Dec;58(6):1001-1007.

11. Rosendorff C, Lackland DT, Allison M, Aronow WS, Black HR, Blumenthal RS, Cannon CP, de Lemos JA, Elliott WJ, Findeiss L, et al. Treatment of hypertension in patients with coronary artery disease: a scientific statement from the American Heart Association, American College of Cardiology, and American Society of Hypertension. J Am Soc Hypertens 2015 Jun;9(6):453-498.

12. National Institute of Health and Clinical Excellence. Hypertension: clinical management of primary hypertension in adults. NICE Clinical Guideline 127. London: NICE; 2011. Accessed 2016 Jun 19. Available from: http://guidance.nice. org.uk/CG127.

13. FDA Prescribing Information. Atenolol and Chlorthalidone Tablets. Accessed 2016 Jun 20. Available from: https://www. drugs.com/pro/atenolol-and-chlorthalidone-tablets.html.

14. Sica DA. Chlorthalidone - a renaissance in use? Expert Opin Pharmacother 2009 Sep;10(13):2037-2039.

15. Pareek A, Basavanagowdappa H, Zawar S, Kumar A, Chandurkar N. A randomized, comparative study evaluating the efficacy and tolerability of losartan-low dose chlorthalidone $(6.25 \mathrm{mg})$ combination with losartan-hydrochlorothiazide $(12.5 \mathrm{mg})$ combination in Indian patients with mild-tomoderate essential hypertension. Expert Opin Pharmacother 2009 Jul;10(10):1529-1536.

16. Carter BL, Ernst ME, Cohen JD. Hydrochlorothiazide versus chlorthalidone: evidence supporting their interchangeability. Hypertension 2004 Jan;43(1):4-9.

17. Ernst ME, Carter BL, Goerdt CJ, Steffensmeier JJ, Phillips BB, Zimmerman MB, Bergus GR. Comparative antihypertensive effects of hydrochlorothiazide and chlorthalidone on ambulatory and office blood pressure. Hypertension 2006 Mar;47(3):352-358.

18. Pareek AK, Messerli FH, Chandurkar NB, Dharmadhikari SK, GodboleAV,KshirsagarPP,AgarwalMA,SharmaKH,MathurSL, Kumbla MM. Efficacy of low-dose chlorthalidone and hydrochlorothiazide as assessed by 24-h ambulatory blood pressure monitoring. J Am Coll Cardiol 2016 Feb 2;67(4):379-389.

19. Khan NA, Campbell NR. Thiazide diuretics in the management of hypertension. Can J Clin Pharmacol 2004 Spring;11(1):e41-e44.

20. Rao SS, Bhagwat AW, Saifi AQ. Mechanism of direct vasodilator effect of thiazide diuretics. Ind J Pharmacol 1981;13(4): 349-351.

21. Zhu Z, Zhu S, Liu D, Cao T, Wang L, Tepel M. Thiazide-like diuretics attenuate agonist-induced vasoconstriction by calcium desensitization linked to Rho kinase. Hypertension 2005 Feb;45(2):233-239.

22. Braunwald E. Heart Disease: a textbook of cardiovascular medicine. 5th ed, W.B. Saunders 1997.

23. MussoMN, VasquezEC,CarvalhinhoFB, BissoliNS, Cabral AM. Chlorthalidone alters the vascular reactivity of DOC-salt hypertensive rats to norepinephrine. Braz J Med Biol Res 1990;23(10):999-1003.

24. Neaton JD, Grimm RH Jr, Prineas RJ, Stamler J, Grandits GA, Elmer PJ, Cutler JA, Flack JM, Schoenberger JA, McDonald R, et al. Treatment of mild hypertension study. Final results. Treatment of Mild Hypertension Study Research Group. JAMA 1993 Aug 11;270(6):713-724.

25. Kostis JB, Cabrera J, Cheng JQ, Cosgrove NM, Deng Y, Pressel SL, Davis BR. Association between chlorthalidone treatment of systolic hypertension and long-term survival. JAMA 2011 Dec 21;306(23):2588-2593.

26. SPRINT Research Group, Wright JT Jr, Williamson JD, Whelton PK, Snyder JK, Sink KM, Rocco MV, Reboussin DM, Rahman M, Oparil S, et al. A randomized trial of intensive versus standard blood-pressure control. N Engl J Med 2015 Nov 26;373(22):2103-2116.

27. American Diabetes Association. Diabetes Care2015;38(Suppl1): S5-S7.

28. Black HR, Davis B, Barzilay J, Nwachuku C, Baimbridge C, Marginean H, Wright JT Jr, Basile J, Wong ND, Whelton P, et al. Metabolic and clinical outcomes in nondiabetic individuals with the metabolic syndrome assigned to chlorthalidone, amlodipine, or lisinopril as initial treatment for hypertension: a report from the Antihypertensive and Lipid-Lowering Treatment to Prevent Heart Attack Trial (ALLHAT). Diabetes Care 2008 Feb;31(2):353-360.

29. Pareek A, Karnik N, Salagre SB, Zawar SD, Joglekar VK, Chandurkar N, Naik GS. Clinical effectiveness of low-dose chlorthalidone $(6.25 \mathrm{mg})+$ atenolol combination in stage I hypertensive patients: a multicenter, randomized, controlled study. Curr Med Res Opin 2008 Jun;24(6):1771-1779.

30. Pareek A, Zawar SD, Salagre SB, Chandurkar NB, Karnik ND. Antihypertensive efficacy of metoprolol XL/low dose chlorthalidone $(6.25 \mathrm{mg})$ combination: a randomized, comparative study in Indian patients with mild-to-moderate essential hypertension. Eur J Med Res 2009 Jul 22;14(7): 297-303.

31. Shafi T, Appel LJ, Miller ER 3rd, Klag MJ, Parekh RS. Changes in serum potassium mediate thiazide-induced diabetes. Hypertension 2008 Dec;52(6):1022-1029. 
32. Barzilay JI, Davis BR, Cutler JA, Pressel SL, Whelton PK, Basile J, Margolis KL, Ong ST, Sadler LS, Summerson J, et al. Fasting glucose levels and incident diabetes mellitus in older nondiabetic adults randomized to receive 3 different classes of antihypertensive treatment: a report from the Antihypertensive and Lipid-Lowering Treatment to Prevent Heart Attack Trial (ALLHAT). Arch Intern Med 2006 Nov 13;166(20):2191-2201.

33. Kortis JB, Wilson AC, Freudenberger RS, et al. Long-term effect of diuretic-based therapy on fatal outcomes in subjects with isolated systolic hypertension with and without diabetes. Am J Cardiol 2005;95:29-35.
34. Barzilay JI, Davis BR, Pressel SL, et al. Long-term effects on incident diabetes mellitus on cardiovascular outcomes in people treated for hypertension. The ALLHAT diabetes extension study. Circ Cardiovasc Qual Outcomes 2012;5:153-162.

35. Preiss D, Seshasai SR, Welsh P, et al. Risk of incident diabetes with intensive-dose compared with moderate-dose statin therapy: a meta-analysis. JAMA 2011;305:2556-2564.

36. Puttnam R, Davis BR, Pressel SL, et al. Association of 3 Different Antihypertensive Medications With Hip and Pelvic Fracture Risk in Older Adults Secondary Analysis of a Randomized Clinical Trial. JAMA Intern Med. doi:10.1001/ jamainternmed.2016.6821. 